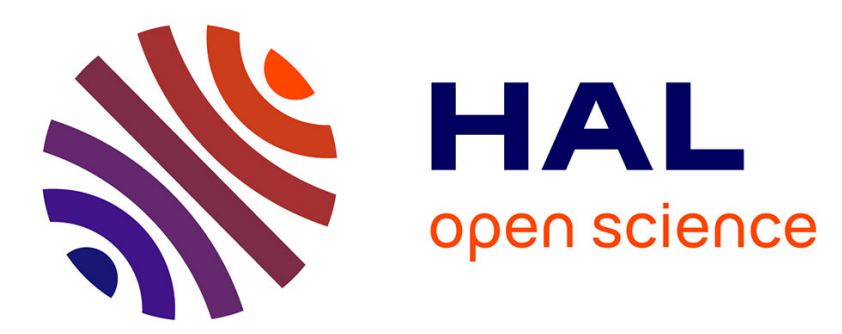

\title{
Characterization of motion cardiac patterns in magnetic resonance cine
}

Fabio Martínez, Antoine Manzanera, Cristina Santa Marta, Eduardo Romero

\section{To cite this version:}

Fabio Martínez, Antoine Manzanera, Cristina Santa Marta, Eduardo Romero. Characterization of motion cardiac patterns in magnetic resonance cine. IEEE International Conference on Image Information Processing, Nov 2011, Shimla, India. pp.1 - 5, 10.1109/ICIIP.2011.6108837 . hal-01119674

\section{HAL Id: hal-01119674 \\ https://hal.science/hal-01119674}

Submitted on 26 Feb 2015

HAL is a multi-disciplinary open access archive for the deposit and dissemination of scientific research documents, whether they are published or not. The documents may come from teaching and research institutions in France or abroad, or from public or private research centers.
L'archive ouverte pluridisciplinaire HAL, est destinée au dépôt et à la diffusion de documents scientifiques de niveau recherche, publiés ou non, émanant des établissements d'enseignement et de recherche français ou étrangers, des laboratoires publics ou privés. 


\title{
Characterization of motion cardiac patterns in magnetic resonance cine
}

\author{
Fabio Martínez ${ }^{1}$, Antoine Manzanera ${ }^{2}$, Cristina Santa Marta ${ }^{3}$, Eduardo Romero $^{1}$ \\ 1 BioIngenium Research Group, Universidad Nacional de Colombia \\ 2 Laboratoire d'Electronique \& Informatique, ENSTA ParisTech \\ 3 Departamento de Física, Matemática y Fluidos, UNED, Madrid- España \\ \{fmartinezc, edromero\}@unal.edu.co, antoine.manzanera@ensta-paristech.fr, cris@dfmf.uned.es
}

\begin{abstract}
One of the most important tasks in Cardiac Magnetic resonance Cine (CMRC) consists in identifying and describing normal and abnormal dynamic heart patterns, a task usually performed by physicians. Segmentation and tracking may support decisions during a particular treatment, but their performance is dependent on the quality of the video. The acquired signal, on the other hand, is contaminated with noise coming from physiological movements and devices, resulting in cardiac blurred boundaries. This paper presents a novel method that automatically identifies flow heart patterns by establishing similarities between two consecutive frames to which a local jet feature analysis has been applied. Once a vector motion field is calculated, spatially connected regions with minimal variance are found as the sources of movement and different statistics objectively estimate movement patterns of these regions. The utility of this method is illustrated by comparing the temporal series of these regions between normal and abnormal patients.
\end{abstract}

Keywords-Local Jet Features, Image motion analysis, magnetic resonance Cine

\section{INTRODUCTION}

Cardiovascular disease (CVD) is an important worldwide health concern that amounts to a $29.2 \%$ of the total global deaths, according to World Health Report in 2003 [1]. Magnetic Resonance Cine (MRC) is a non invasive video technique that provides cardiac anatomic information with good spatial and temporal resolution, allowing the analysis of the heart dynamic patterns. These analyses aim to estimate, globally, the myocardial function, and specifically, the wall motion information. Therefore they are used as an indicator of the pathological and normal movements [2]. Overall, these analyses are carried out by expert physicians, whereby results are highly subjective. Likewise, in the actual clinical routine there is no objective measurement upon the cardiac flow, a fundamental limitation of this analysis. This may be especially useful in early disease detection, case in which the boundary between normal and abnormal is quite diffuse, even for expert physicians. The point is that any objective measurement must take into consideration different variation sources, namely, changes of pose and reflection properties of the object, acquisition device noise and similar tissue properties of the neighboring heart tissues. For instance, in the video, it is really difficult to establish a neat border between heart and liver [3, 4].
So far different approaches have been proposed to obtain dynamic and structural heart patterns. Some of them, based on region growing or edge-detection, present difficulties when dealing with noise, grey scale variations and low gradients. Therefore, a high degree of supervision is required [5]. On the other hand, other works have used MR tagged images, a technique in which the tissue motion is related to the tag line locations or intersections [6]. Active contours or statistical models that attempt to track the ventricle movements have been also used. These active contours are computationally expensive and their performance in noisy conditions is very poor. The statistical models are not correlated with any physiological phenomenon, a very important issue from the clinical standpoint since the objective is not uniquely to follow a movement but rather to establish patterns with physiological meaning [7, 8]. Finally, even if the tagging technique offers appropriate quantitative analysis for some dynamic parameters, its clinical use has so far remained limited mainly because its performance is very poor when the acquisition process is noisy. Besides, the tagging resolution is so low that the physiological meaning of the found flow patterns is really difficult to establish [8]. 
(a)

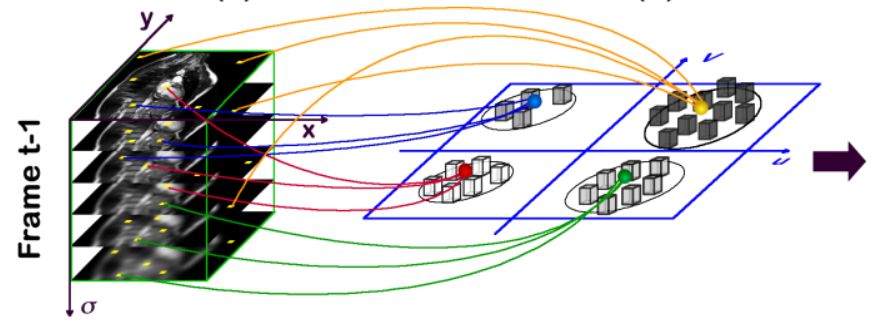

(c)

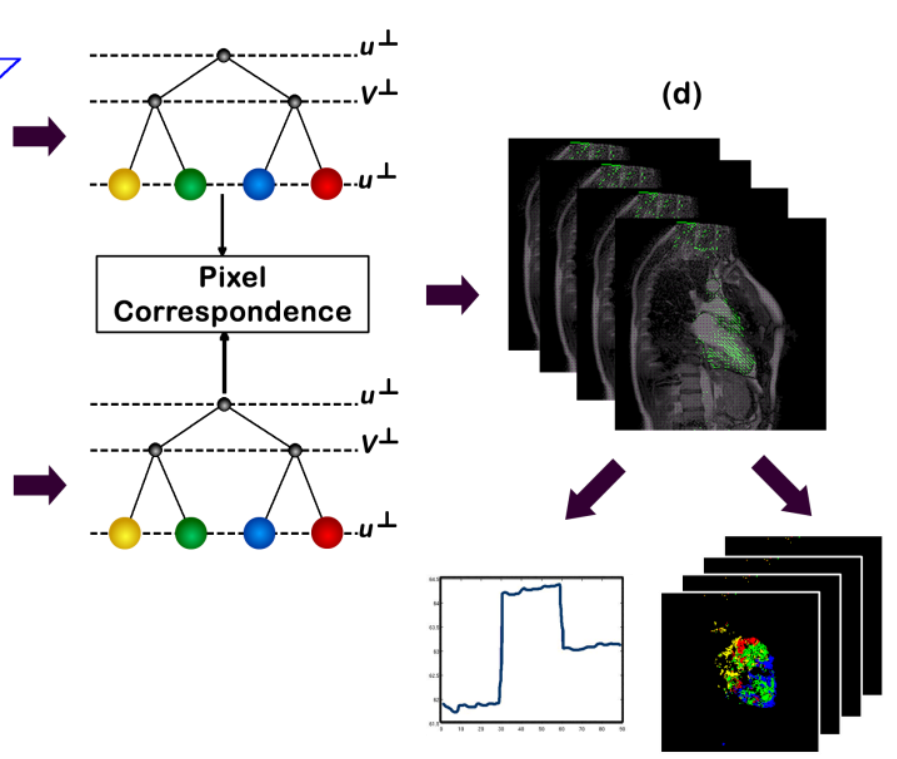

(e)

Figure 1: Local jet features from MRC slices are calculated and stored in a kd-tree by similarity. The motion is estimated by searching a particular pixel in the precedent kd-tree. Once the apparent heart motion is obtained, some metrics define the dynamic patterns.

The main contribution of this work is the design of a fully automatic method which determines the fluid vector field that represents the apparent heart motion. The method allows to define objective measurements upon the main FOE (Foci of Expansion), defined as those locations with minimal motion from which line fields are coming out from one of these foci to another. This paper is organized as follows: We first briefly present the dataset, then Section III introduces the proposed method, Section IV demonstrates the effectiveness of the method and the proposed metrics, a useful tool to help the expert physician. The last section concludes with a discussion and possible future works.

\section{DATASET DESCRIPTION}

Ten different cases were herein included to assess the proposed method, five normals, three diagnosed with Fallot tetralogy and the last two, with acute myocardial infarction. The images were acquired in a $1.5 \mathrm{~T}$ Intera scanner (Philips Medical Systems, The Netherlands), equipped with $30 \mathrm{mT} / \mathrm{m}$ gradients, using a dedicated 5 elements phased array cardiac coil for every patient, The 4D sequences follow the conventional views: short axis, two chambers and four chambers. A balance gradient echo sequence (balanced TFE) has been used in all cases. The most relevant parameters for the short axis view are: slices $=13$, slice thickness $=8 \mathrm{~mm}$, heart phases $=30, \mathrm{TR} / \mathrm{TE}=3.15 \mathrm{~ms}$, flip angle $=60^{\circ}$, matrix $=$ $256 * 192$, SENSE factor $=1.5$, turbo factor $=8$. For the two chambers view: slices $=2$, slice thickness $=8 \mathrm{~mm}$, heart phases $=20, \mathrm{TR} / \mathrm{TE}=2.7 / 1.3 \mathrm{~ms}$, flip angle $=60^{\circ}$, matrix $=$
$256 * 186$, SENSE factor $=1.5$, turbo factor $=24$. Finally, for the 4 chambers view: slices $=2$, slice thickness $=8 \mathrm{~mm}$, heart phases $=20, \mathrm{TR} / \mathrm{TE}=3.2 / 1.6 \mathrm{~ms}$, flip angle $=60^{\circ}$, matrix $=$ $256 * 175$, SENSE factor $=1.5$, turbo factor $=15$.

\section{THE PROPOSED METHOD}

The whole method starts by calculating the Jacobian and Hessian matrices for different scales of a slice, using a Local Jet Feature approach [9]. The resulting local jet representation is then grouped by similarity i.e., foci with minimal variance. A kd-tree structure stores the values of local jet for every pixel. The motion is estimated by searching a particular pixel in the precedent kd-tree (the kd-tree at time $t-1$ ). Such search is firstly addressed to the pixel with the closer local jet feature Euclidean distance. The kd-tree structure allows orientating this search to the pixels with similar local feature description. Once this most similar pixel is found, the two associated coordinates are used to calculate the motion vector. One main contribution of the present work was the design of an objective measurement on the obtained vector field: spatially connected regions with minimal variance were firstly grouped, i.e. regions that maximize the inter-group variance regarding the vectors magnitudes and angles. 

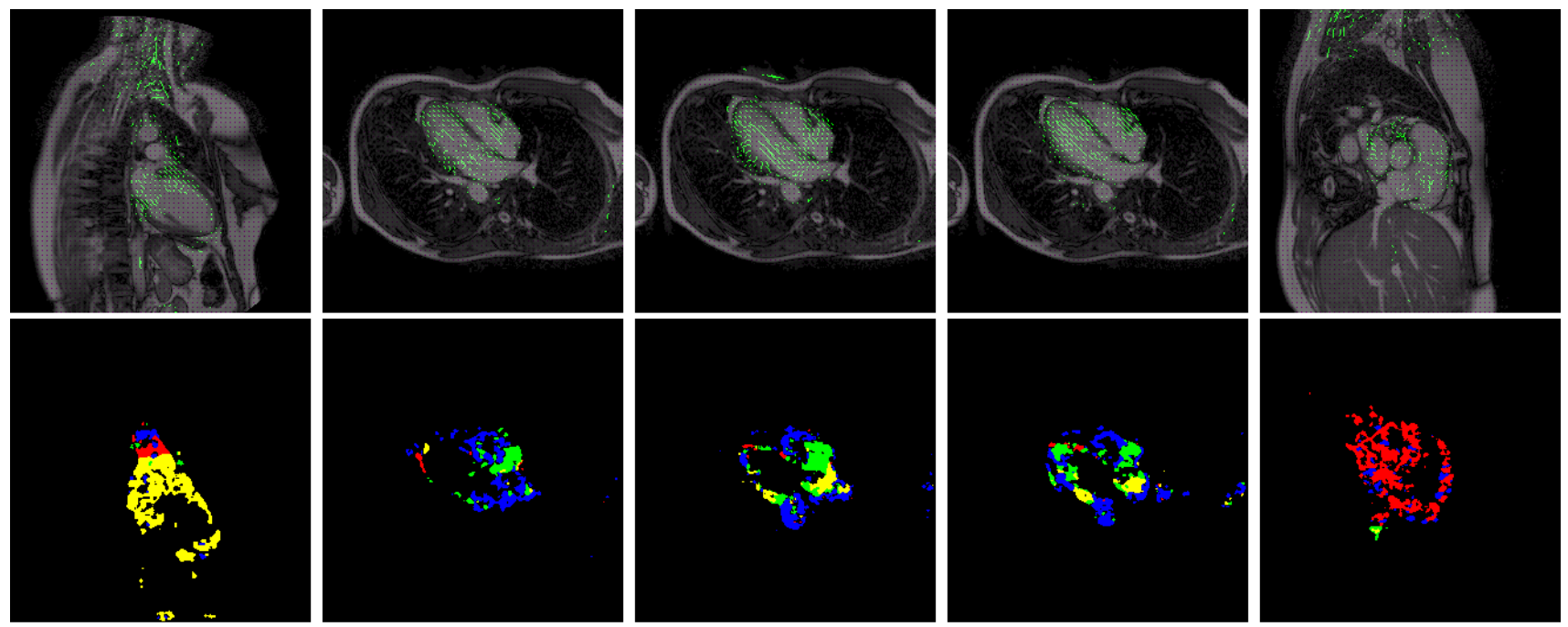

Figure 2: The flow determined by the different selected clusters on different slices of a cardiac cycle.

Different statistics were calculated on the obtained regions. The apparent heart motion was thus estimated by grouping up spatial locations with similar variance, as illustrated in Figure 1 .

\section{A. $\quad$ The Multiscale Local Jet Similarity Space}

Nowadays it is well accepted in the computer vision community that relevant information can be determined by different strategies that measure correlation or coherence through different scales $[10,11]$. The strategy herein applied is known as local jets and basically consist in obtaining an homogenous partition of the frequential spectrum by analyzing the image at different scales, which are then characterized by a map of partial derivatives of different order (we used here a first and second orders but the analysis can be extended to higher orders when one might want a finer spectral partition). In consequence, the first step in our approach was to obtain a collection of spatial derivates at different scales from independent slices, as follows

$$
F_{i j}^{\sigma}=f * \frac{\partial^{i+j} G_{\sigma}}{\partial x_{1}^{i} \partial x_{2}^{j}}
$$

where $G_{\sigma}$ is the 2 d Gaussian function with standard deviation $\sigma$.

The Gaussian kernel is here justified because this is the unique kernel with a homogeneous scale-space representation (linearity and shift invariance in both frequency and space) [10]. The multi-scale local jet is given by the collection $\left\{f_{i j}^{\sigma} ; i+j \leq r, \sigma \in S\right\}$, where $\mathrm{r}$ is the derivation order and $\mathrm{S}=$ $\sigma_{1}, \cdots, \sigma_{\mathrm{q}}$ the selected scales.

Local jets as descriptors have been already used on natural images, in which it has been shown that the first eigen (singular) vectors obtained by PCA or SVD are quite similar to the first derivatives of a $2 \mathrm{~d}$ Gaussian function [12, 13], whereby this representation is quite sparse. Therefore, information can be automatically estimated by calculating the energy concentrated at each scale.

Then, local jets are normalized [10]:

$$
F_{i j}^{\sigma}=\frac{\sigma^{i+j}}{i+j+1} f_{i j}^{\sigma}
$$

Where $\sigma^{i+j}$ is the scale normalization factor and $i+j+1$ is the number of $(\mathrm{i}+\mathrm{j})$-order derivatives. The following single scale distance is used:

$$
d_{f}^{\sigma}(x, y)=\sum_{i+j \leq r}\left(F_{i j}^{\sigma}(x)-F_{i j}^{\sigma}(y)\right)^{2}
$$

\section{B. Data structure}

Once a MRC slice is represented by a multi-scale set of features (local jet collection $F_{i j}$ ), the next step consists in storing the local jet in a kd-tree structure $\mathrm{F}$. This data structure, a binary space partition, organizes the whole set of features and allows rapid access to them [14], performing nearest neighbor (NN) searches in the feature space. This efficient search is extensively used to perform operations based on similarity between pixels that are not connected in the image space. 


\section{Heart Motion Estimation}

The apparent heart motion is estimated for every pixel $x$ at frame $t$ by using the similarity cluster established before. At frame $t$, slice $f_{t}$, and pixel $x$, we compute $u\left(f_{t-1}, f_{t}, x\right)$, the nearest neighbor of the feature vector associated to $\mathrm{x}$ in the $f_{t-1}$ feature space:

$$
u\left(f_{t-1}, f_{t}, x\right)=\arg \operatorname{mín}_{v \in \mathcal{F}_{f_{t-1}}} d^{F}\left(\hat{x}_{f_{t}}, v\right)
$$

Where $\hat{\mathrm{x}}_{\mathrm{ft}}$ is the $x$ projection in the feature space of $f$, i.e., $\hat{x}_{f_{t}}=\left\{f_{i, j}(x)\right\}$, and $d^{F}$ denotes the feature space distance. Then we compute $y\left(f_{t-1}, f_{t}, x\right)$, the pixel from $f_{t-1}$ which is the most similar to $x$ from $f_{t}$ :

$$
y\left(f_{t-1}, f_{t}, x\right)=\underset{z \in \mathcal{F}_{f_{t-1}^{-1}}^{-1}}{\arg } \min _{\left(f_{t-1}, f_{t}, x\right)} d^{I}(x, z)
$$

where $d^{I}$ denotes the distance in the image space.

We obtain the pixel from the set of pixels associated to the feature vector $\mathrm{u}$ which is the closest to $\mathrm{x}$ in the image space. Finally, the velocity vector is computed as the difference:

$$
c\left(f_{t-1}, f_{t}, x\right)=x-y\left(f_{t-1}, f_{t}, x\right)
$$

Our method was implemented in $\mathrm{C}++$ language on a standard PC (AMD with $2.66 \mathrm{GHz} \mathrm{CPU}$ and $3 \mathrm{~GB}$ of RAM) takes about $10-15 \mathrm{sec}$ to process all slices of a plane.

\section{Computed Measures}

Our fundamental hypothesis is that those places with minimal movement constitute a stable source of information of the whole flow pattern. Once the vector flow was determined, the next measurements were proposed:

- Flow motion clustering: Similar flows are grouped as the sets of points with minimal variance regarding the vector magnitude and angle. The number of sets depends on the type of description one might want to obtain, for instance with $\mathrm{k}=2$ the two circulatory circuits are repaired, i.e., left and right. With $\mathrm{k}=4$ we observe the principal fluid directions, mostly in the left part of the circulatory circuit. A larger number produces more clusters but with no physiological meaning.

- Global and local statistic measures: computing simple statistics either globally or in a small neighborhood, results in a saliency map of the flow pattern. This saliency map corresponds to heart segmentation, when this is locally considered, and to a tracking of the Center of mass, when the analysis is global. In both cases, these patterns (as it will be illustrated later) show very different appearance.

\section{EXPERIMENTAL RESULTS}

The method parameters were adjusted to obtain the best performance and then set to the following values: $\mathrm{NN}=1$ with a local jet order of 2 and $\sigma=5$. Typical results of computing motion estimations for the three planes are shown in Figure 2(upper sequence). After visual evaluation, the expert determined that the proposed method achieved a very realistic motion simulation during a cardiac cycle. Figure 2 (lower sequence) shows the flow determined by the different selected clusters on every slice of a cardiac cycle. On the other hand, it is possible to use the saliency map built up from the computed flow (magnitude and norm) and determine a center of mass of this flow or global heart trajectory, as showed in Figure 3. This trajectories can be used as dynamic patterns to identify or grouping different kinds of movement. The RMSE computed from global trajectories demonstrated a minimal error between similar movements as illustrated in Table 1, for instance, the difference among normal flows is 0.04 in average, while the difference with Fallot tetralogy is 20.51 and the difference with infarction movement is 23.023. This difference suggests that this measure could be used as indicator of some abnormal movements.

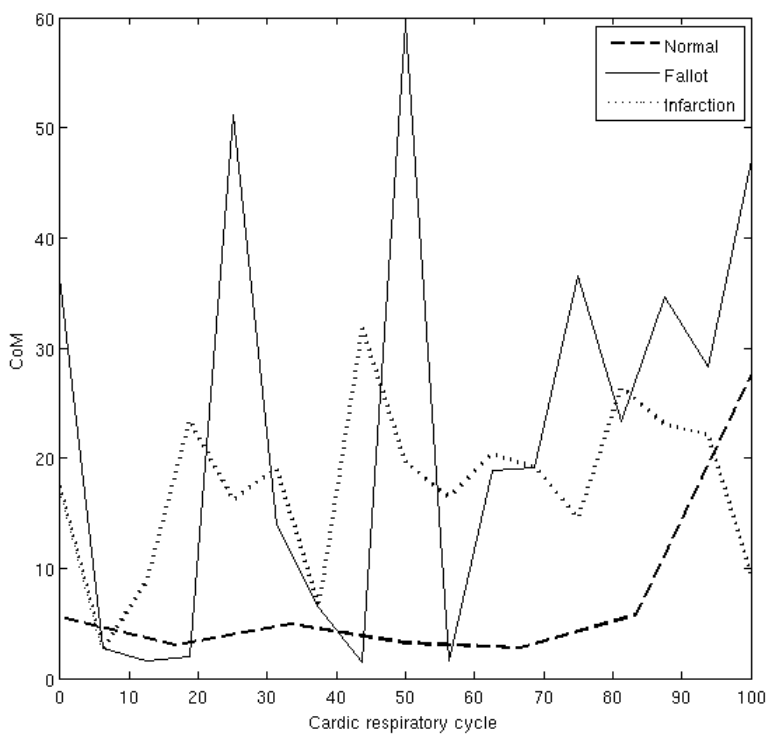

Figure 3: Mean trajectories of CoM heart movement computed since the saliency map. In this figure it is possible to observe the characteristic of global motion computed from saliency map and the strong differences between the three kinds of movements.

TABLE I. Root Mean Squared Error for the three evaluated movements. These averages have been calculated from the differences between the signals and the mean representative signal to every movement of the cardiac cycle.

\begin{tabular}{|l|c|c|c|}
\hline Mean Signal & $\begin{array}{l}\text { RMSE } \\
\text { Normal }\end{array}$ & $\begin{array}{l}\text { RMSE } \\
\text { Fallot }\end{array}$ & $\begin{array}{l}\text { RMSE } \\
\text { Infarction }\end{array}$ \\
\hline $\begin{array}{l}\text { Mean } \\
\text { Normal }\end{array}$ & 0.04 & 20.57 & 23.16 \\
\hline Mean Fallot & 20.51 & 3.89 & 10.27 \\
\hline $\begin{array}{l}\text { Mean } \\
\text { Infarction }\end{array}$ & 23.023 & 8.81 & 3.82 \\
\hline
\end{tabular}




\section{CONCLUSIONS}

We have introduced a new method to analyze the heart dynamics by estimating the apparent motion. We obtain a robust representation which captures some important heart dynamic and static features, for instance the most important topological elements that represent both places with minimum coherent movement (fluid sources) and with largest change rate (they represent the fluid lines). Experiments demonstrated that this can be used to characterize fluid patterns and establish a base normal line, as confirmed by an expert in the domain. Measures presented in this work constitute a first approximation to understanding the complex dynamic heart. From this kind of analyzes, we expect that it is possible to set up a collection of descriptors which allow to accurately describe motions patterns and quantify its semantics. The presented procedure could be extended to other type of medical imaging problems, or may be used to segment the wall of the heart with better accuracy by taking advantage of the temporal information. On the other hand, the combination of our method with statistical approaches would allow tracking of specific heart areas.

\section{REFERENCES}

[1] World Health Organization, "World health report 2003," 2003.J. Clerk Maxwell, A Treatise on Electricity and Magnetism, 3rd ed., vol. 2. Oxford: Clarendon, 1892, pp.68-73.

[2] A. Hennemuth, A. Seeger, O. Friman, S. Miller, B. Klumpp, S. Oeltze, and H.-O. Peitgen, "A comprehensive approach to the analysis of contrast enhanced cardiac mr images," Medical Imaging, IEEE Trans on, vol. 27, no. 11, pp. $1592-1610,2008$.
[3] N Paragios, "A level set approach for shape-driven segmentation and tracking of the left ventricle," IEEE Trans on Medical Imaging, vol. 22, pp. $773-776,2003$.

[4] M. Joll, C. Guetter, and J. Guehring, "Cardiac segmentation in mr cine data using inverse consistent deformable registration," ISBI, 2010.

[5] M. Lynch, O. Ghita, and P.F. Whelan, "Automatic segmentation of the left ventricle cavity and myocardium in mri data," Computers in Biology and Medicine, vol. 36, pp. 389 - 407, 2006.

[6] D.L. Kraitchman, A.A. Young, Cheng-Ning Chang, and L. Axel, "Semiautomatic tracking of myocardial motion in mr tagged images," Medical Imaging, IEEE Trans on, vol. 14, no. 3, pp. 422-433, Sept. 1995

[7] M.A. Guttman, J.L. Prince, and E.R. McVeigh, "Tag and contour detection in tagged $\mathrm{mr}$ images of the left ventricle," Medical Imaging, IEEE Trans on, vol. 13, no. 1, pp. 74-88, Mar. 1994.

[8] Ihor Smal, Wiro Niessen, and Erik Meijering, "Particle filtering methods for motion analysis in tagged mri," ISBI, 2010.

[9] B. Florack, L. Ter Haar Romeny, M. Viergever, and J. Koenderink, "The gaussian scale-space paradigm and the multiscale local jet," International Journal of Computer Vision, vol. 18, pp. 61-75, 1996, 10.1007/BF00126140.

[10] T. Lindeberg, "Feature detection with automatic scale selection," Int. J. Comput. Vision, vol. 30, no. 2, pp. 79-116, 1998.

[11] B.M. Romeny, Front-End Vision and Multi-Scale Image Analysis: Multi-scale Computer Vision Theory and Applications, written in Mathematica, Springer Publishing Company, Incorporated, 2009

[12] J. Orchard, M. Ebrahimi, and A. Wong, "Efficient nonlocalmeans denoising using the svd," 2008, pp. 1732-1735.

[13] A. Manzanera, "Local jet based similarity for nl-means filtering," Pattern Recognition, International Conference on, pp. 2668-2671, 2010.

[14] Jon Louis Bentley, "Multidimensional binary search trees used for associative searching," Commun. ACM, vol. 18, no. 9, pp. 509-517, 1975.

[15] Sunil Arya, David M. Mount, Nathan S. Netanyahu, Ruth Silverman, and Angela Y.Wu, "An optimal algorithm for approximate nearest neighbor searching fixed dimensions," J. ACM, vol. 45, no. 6, pp. 891923, 1998. 